\title{
Atopic Predilection among Kawasaki Disease Patients: A Cross-Sectional Study of 1,187,757 Teenagers
}

\author{
Ayal Hassidim ${ }^{a}$ Ilan Merdler ${ }^{b}$ Odelia Chorin $^{d}$ Rona Merdler-Rabinowicz ${ }^{\mathrm{e}}$ \\ Ilan Dallal ${ }^{d}$ Maxim Perlman ${ }^{f}$ Ehud Chorin $^{c}$ \\ a Department of Plastic and Reconstructive Surgery, Hadassah-Hebrew University Medical Center, Jerusalem, \\ Departments of ${ }^{\mathrm{b}}$ Internal Medicine $\mathrm{H}$ and ${ }^{\mathrm{C} C a r d i o l o g y}$, Tel Aviv Sourasky Medical Center (affiliated to the Sackler \\ School of Medicine, Tel Aviv University), Tel Aviv, ${ }^{d}$ Department of Pediatrics, E. Wolfson Medical Center (affiliated \\ to the Sackler School of Medicine, Tel Aviv University), Holon, and e Department of Pediatrics, Sheba Medical Center \\ and ${ }^{\mathrm{f} I s r a e l i}$ Defense Forces, Medical Corps, Tel Hashomer, Israel
}

\section{Key Words}

Kawasaki disease - Allergic rhinitis - Asthma - Angioedema · Urticaria

\begin{abstract}
Background: Kawasaki disease (KD) is an acute, systemic vasculitis in children, with an etiology that is not completely understood. It is assumed that the development of KD is mediated by an immunologic response. Several reports from East Asia have found a higher prevalence of atopic diseases among patients with KD, but a large-scale study of a nonAsian population regarding this correlation is still lacking. The purpose of this article was to achieve this goal. Methods: We conducted a cross-sectional, large-scale study to estimate the correlation of KD with allergic diseases. The medical history of 1,187,757 Israeli teenagers (aged 16-20 years during the years 1998-2013) was retrieved. The study population was divided into 3 groups according to a past history of noncomplicated and complicated KD and a control group. The prevalence of allergic diseases among these groups was further investigated. Results: The prevalence of atopic diseases in the 3 study groups was presented (asthma in 11.4,
\end{abstract}

8.1 and $3.5 \%$, respectively; angioedema/urticaria in $7.1,0$ and $0.46 \%$, respectively; allergic rhinitis in 20, 12.1 and $6.7 \%$, respectively). In noncomplicated $\mathrm{KD}$, a statistically significant link to asthma [odds ratio (OR) 2.4; $p=0.048$ ] and a borderline significant link to allergic rhinitis (OR 1.9; $p=0.06$ ) were found. In KD complicated with cardiac disease, statistically significant links were found for all the allergic conditions, asthma (OR 3.5; $p=0.003$ ), allergic rhinitis (OR 3.5; $\mathrm{p}<0.001$ ) and angioedema/urticaria (OR 16.48; $\mathrm{p}<0.001$ ). Conclusion: KD is associated with allergic diseases. This association increases with the severity of the disease.

(c) 2016 S. Karger AG, Basel

\section{Introduction}

First described in 1967, Kawasaki disease (KD) is an acute, systemic vasculitis that occurs predominantly among children aged $\leq 5$ years [1]. It affects the blood vessels, skin, mucous membranes and lymph nodes. The

Ayal Hassidim and Ilan Merdler contributed equally to this work.

\section{KARGER}

E-Mail karger@karger.com

www.karger.com/iaa
C 2016 S. Karger AG, Basel

$1018-2438 / 16 / 1702-0092 \$ 39.50 / 0$
Correspondence to: Dr. Ilan Merdler

Department of Internal Medicine H, Tel Aviv Sourasky Medical Center Weizman St. 6

Tel Aviv 64239 (Israel)

E-Mail ilanmerdler@gmail.com 
skin and mucous membranes become red and inflamed, the hands and feet swell, lymph nodes in the neck are often enlarged, and there is recurrent fever. Coronary artery aneurysms or ectasia develop in $15-25 \%$ of children with untreated disease and may lead to myocardial infarction, sudden cardiac death or ischemic heart disease, comprising the most deleterious complications [13]. In fact, $\mathrm{KD}$ is now the leading cause of acquired heart disease among pediatric populations in the developed world $[2,3]$.

The precise etiology of KD remains unknown, although clinical and epidemiological features, such as the wave-like, geographic spread with community outbreaks, strongly suggest a transmittable childhood infectious cause. However, efforts to identify an infectious agent with conventional bacterial and viral cultures or serological methods, as well as with animal inoculation, have failed $[4,5]$. It may be that $\mathrm{KD}$ results from an immunologic response that is triggered by any of several different and yet unidentified microbial agents [6-9].

An alternative leading hypothesis involves a ubiquitous infectious agent that produces clinically apparent disease only in certain genetically predisposed individuals. In Israel, the estimated incidence of KD is $11.9 / 100,000$ [10] in children $<5$ years of age, with a male-to-female ratio of 1.7:1, and a peak incidence in winter and spring. Among Asian populations, the incidence reported is much higher, ranging from 69 to 239/100,000 [6]. A hypothesis involving genetic predisposition is supported by the increased incidence of the disease among these populations, also when compared to European and American populations, where the estimated incidence rates are 5.8/100,000 [11].

Reports from East Asia have found a higher prevalence of atopic dermatitis, allergic rhinitis and asthma among children diagnosed with $\mathrm{KD}$ compared to the normal population [12-16]. However, to the best of our knowledge, large-scale studies regarding the incidence of allergic diseases occurring in KD patients are still lacking, especially regarding non-Asian populations.

The goal of this study was to evaluate the prevalence of allergic diseases among a heterogenic population of Israeli adolescents diagnosed with $\mathrm{KD}$.

\section{Methods}

We conducted a cross-sectional study to estimate the epidemiologic relationship between KD and allergic diseases.
Source of Data

As part of the military preconscription assessment, most Israeli civilians undergo a routine medical evaluation and fitnessfor-service classification at a prespecified age (usually 17 years). The medical evaluation includes questionnaires filled in by the candidate and his/her family practitioner as well as further tests and consultations as required. The result of this process is $\geq 1$ fitness-for service (FFS) classification numerical codes indicative of a medical diagnosis.

From the conscripts' medical records, we retrieved demographic details and FFS codes. The presence of $\mathrm{KD}$ was determined by a corresponding numerical code and graded as complicated/ noncomplicated. The meaning of complicated KD is that there was any kind of cardiac manifestation. The presence of asthma, allergic rhinitis and angioedema or chronic urticaria was also determined. Patients with chronic urticaria were assigned the same code as angioedema. We must emphasize that the group of patients with either angioedema or urticaria is problematic. These individuals were grouped together for technical reasons, and, unfortunately, we could not separate them into 2 groups. There were no data regarding atopic dermatitis.

The data were based on family practitioners' declarations of KD in the past. The data were gathered when the subjects were 17 years old, without knowledge, however, of their exact age when they had the illness. In addition, these same declarations were used in order to gather data regarding cardiac manifestations. We only had data according to the questionnaires regarding nonspecific cardiac manifestations without any further details. We must emphasize that the distinction between KD with or without cardiac manifestations was done retrospectively and without enough data as to the nature of the complications, medical therapy or timing. We still included the distinction in the study because we thought it was important to examine the data for each group separately, the assumption being that most cardiac manifestations were coronary aneurysms (without noting the medical treatment).

The study was approved by the Israeli Defense Forces' (IDF) institutional review board, and the subjects' anonymity was strictly kept. Socioeconomic status (SES) was one of the demographic features and was graded on a scale of 1-10 (lowest to highest).

\section{Study Population}

The study population included all Israeli teenagers who underwent medical evaluation by the IDF as part of the preconscription assessment during the years 1998-2013. We excluded all subjects who were younger than 16 years or older than 20 years at their medical evaluation.

\section{Statistical Analysis}

All data were summarized and displayed as mean $( \pm S D$ when applicable) for continuous variables and as number (or \%) of patients in each group for categorical variables. Continuous variables were compared using the independent-samples t test or ANOVA (the Scheffé test was used for post hoc multiple comparisons) while categorical variables were compared using the $\chi^{2}$ test and binary logistic regression models. Based on the presence of the corresponding FFS code, we divided the study population into 2 groups, i.e. cases (of the respective allergic condition tested) and controls (general population). We then divided both cases and controls into 2 groups of $\mathrm{KD}$ cases and controls in a similar manner, yielding a $2 \times 2$ table. We calculated the odds ratio (OR) for the presence of 
Table 1. Characteristics and prevalence of diseases in the study cohort

\begin{tabular}{|c|c|c|c|c|}
\hline Variable & $\begin{array}{l}\text { Complicated } \\
\text { KD }\end{array}$ & $\begin{array}{l}\text { Noncomplicated } \\
\text { KD }\end{array}$ & $\begin{array}{l}\text { General } \\
\text { population }\end{array}$ & p value ${ }^{1}$ \\
\hline Subjects, $\mathrm{n}$ & 70 & 74 & $1,187,757$ & \\
\hline Males, \% & 78.5 & 71.6 & 58.5 & 0.031 \\
\hline Mean (SD) SES & $6.77(1.553)$ & $6.31(1.743)$ & $5.8(1.675)$ & 0.036 \\
\hline Mean (SD) BMI & $22.2(3.376)$ & $22.62(3.989)$ & $22.11(3.92)$ & 0.267 \\
\hline Asthma prevalence, $\%$ & 11.4 & 8.1 & 3.55 & 0.048 \\
\hline Angioedema/chronic urticaria prevalence, $\%$ & 7.1 & 0 & 0.46 & 1 \\
\hline Allergic rhinitis prevalence, $\%$ & 20 & 12.1 & 6.7 & 0.06 \\
\hline
\end{tabular}

${ }^{1}$ Calculated with a t test for the noncomplicated KD group versus the general population group.

Table 2. OR of cases of KD and allergic conditions compared to the general population group

\begin{tabular}{lccc}
\hline & OR & CI & p value \\
\hline $\begin{array}{l}\text { Noncomplicated KD } \\
\text { Asthma }\end{array}$ & 2.391 & $1.038-5.51$ & 0.048 \\
Angioedema/chronic urticaria & 0.995 & $0.995-0.995$ & 1 \\
Allergic rhinitis & 1.918 & $0.955-3.852$ & 0.06 \\
\hline Complicated KD & & & \\
Asthma & 3.497 & $1.675-7.304$ & 0.003 \\
Angioedema/chronic urticaria & 16.48 & $6.634-40.941$ & $<0.001$ \\
Allergic rhinitis & 3.464 & $1.928-6.222$ & $<0.001$ \\
\hline All KD patients & & & \\
Asthma & 2.919 & $1.682-5.067$ & 0.001 \\
Angioedema/chronic urticaria & 7.706 & $3.156-18.813$ & 0.001 \\
Allergic rhinitis & 2.634 & $1.686-4.114$ & $<0.001$ \\
\hline
\end{tabular}

the tested allergic condition between $\mathrm{KD}$ cases and controls. Confidence interval (CI) and statistical significance were calculated for the OR after adjusting for age and gender. A 2-tailed $p<0.05$ was considered statistically significant. All analyses were performed with SPSS v21.0 software (SPSS Inc., Chicago, Ill., USA).

\section{Results}

\section{Study Population}

We examined the computerized military medical data of 1,187,757 Israeli adolescents for the years 1998-2013. Of these, $144(0.01 \%)$ had KD, 74 of whom were classified as noncomplicated and 70 as complicated (table 1).

Significant differences were found in the demographic details of the KD group in comparison to the general population (controls). There was a larger percentage of males,
78.5 and $71.6 \%$ in the complicated and noncomplicated $\mathrm{KD}$ groups, respectively, compared to $58.5 \%$ in the general population. The SES was significantly higher in the KD groups, and a larger proportion of the KD groups was born in Israel. No statistically significant differences were found with regard to $\mathrm{BMI}$ in comparison to the general population.

\section{Prevalence of Atopic Disease in the Study Cohort}

The prevalence of asthma was higher in the KD group, 11.4 and $8.1 \%$ in the complicated and noncomplicated groups, respectively, in comparison to $3.55 \%$ in the general population. The prevalence of angioedema or urticaria was higher in the complicated KD group at $7.1 \%$, in comparison to $0.46 \%$ in the general population. The prevalence of allergic rhinitis was $20 \%$ in the complicated KD group, $12.1 \%$ in the noncomplicated $\mathrm{KD}$ group and $6.7 \%$ in the general population. 
Association of Various Allergic Conditions with KD

We examined the link between asthma, angioedema or allergic rhinitis and noncomplicated and complicated $\mathrm{KD}$ and compared it to the general population (table 2). In cases of noncomplicated $\mathrm{KD}$, a significant link to asthma was discovered (OR 2.391; $\mathrm{p}=0.048)$, and a nearly significant link to allergic rhinitis (OR 1.9; $\mathrm{p}=0.06$ ). No significant link was discovered between noncomplicated KD and angioedema/chronic urticaria. In the cases classified as complicated $\mathrm{KD}$ with cardiac complications, a statistically significant link was discovered to all the allergic conditions that were examined, i.e. asthma (OR 3.5; p = 0.003 ), allergic rhinitis (OR 3.5; $\mathrm{p}<0.001$ ) and angioede$\mathrm{ma} /$ chronic urticaria (OR 16.48; $\mathrm{p}<0.001$ ).

\section{Discussion}

In this study, we evaluated the association between $\mathrm{KD}$ and allergic conditions in a large non-Asian population. The main findings of our analysis are: (1) the prevalence of KD in our cohort was 12.1/100,000, (2) SES was higher among KD patients in comparison to the general population, (3) there was a higher prevalence of atopic diseases among individuals with a history of $\mathrm{KD}$, and (4) the link between $\mathrm{KD}$ and atopic diseases was more significant among the complicated $\mathrm{KD}$ cases.

The major strength of this study is the large and mostly unbiased study population forming the basis for the data we retrieved. Its weakness is the fact that mostly questionnaire data were used to characterize the clinical course of the patients. This can lead to a possible bias. We had no information on the type of KD cardiac complication, medical treatment or timing.

The association between KD and atopic diseases has been proven before, but for Asian populations only. Our study is the first to reveal that KD is linked to allergic diseases in a non-Asian population as well. In previous studies from Taiwan, Tsai et al. [12] found that KD patients aged $0-13$ years had a higher prevalence of asthma, urticaria and allergic rhinitis. Kuo et al. [13] reported an increased risk for atopic diseases among KD patients, with the highest hazard ratio being 1.5 among asthma patients; however, the OR reported by Liew et al. [14] is more similar to our findings at 3.75 for any allergy among $\mathrm{KD}$ patients. Our higher OR could be attributed to the lack of chronological insight as well as to the large sample size.

We further proved that this link is associated with the severity of $\mathrm{KD}$, as complicated $\mathrm{KD}$ patients were more prone to allergic diseases compared to noncomplicated KD patients. To the best of our knowledge, this is the first study to examine the link to allergic diseases among subjects with the noncomplicated and the complicated forms of KD.

Since our study was designed as a cross-sectional study, we cannot determine whether the KD preceded the atopic disease or vice versa. Webster et al. [15] showed that atopic tendency existed prior to the onset of $\mathrm{KD}$ and was also persistent among family members of KD patients. Wei et al. [16] reported the OR for developing KD among atopic patients. In their study, patients with urticaria had the highest risk, followed by patients with allergic rhinitis and atopic dermatitis (OR 1.8, 1.44 and 1.22, respectively).

The underlying etiology or mechanism for this association between $\mathrm{KD}$ and atopic disease is not completely understood. Despite intensive studies, the analysis of T-cell functions in KD has shown variable and often conflicting results [16-18]. Th1 cells activate macrophages and cytotoxic $\mathrm{T}$ cells while Th2 cells initiate humoral immunity and allergic inflammation. Th1 and Th2 have a reciprocal effect on each other. There is still not enough information regarding a possible mechanism related to T-cell activation that links the inflammatory process in KD to allergies. Studies exploring the immune system of KD patients have reported mutations in the mannose-binding lectin gene, a part of the innate immune system, as holding a potential role for developing $\mathrm{KD}[19,20]$. Of note are the increased levels of $\operatorname{IgE}$ and eosinophilia that were found in the peripheral blood of KD patients [17, 21].

Matsuoka et al. [18] investigated environmental risk factors for developing allergic diseases, and found higher rates of smoking among KD patients which can predispose patients to allergic diseases. However, the paradox reported in the same study was the higher rates of household pets among KD patients, which is thought to be a protective factor against asthma according to the 'hygiene hypothesis'.

Other studies reported increased levels of matrix metalloproteinase in both asthma and KD patients [22, 23]. There is also a possible bias regarding health-seeking patterns among KD patients compared to the general healthy population, which may render overdiagnosis of allergic diseases when making comparisons.

Our study has several limitations. Most were mentioned earlier in this paper. The main limitation is that data were based on personal questionnaires and reviewing of past medical records, so inaccurate data may have led to a possible misclassification of the relevant diseases. However, since we used a large database, it is reasonable to assume that this limitation would have only a minor effect on the results. 
As discussed above, based on our cross-sectional study, we could not ascertain whether allergy is a longterm complication of $\mathrm{KD}$ or a prior phenomenon with an established link. Furthermore, we have no data regarding the treatments that were given for the KD patients, and the potential effects these might have for developing allergic diseases. We could also not differentiate the link to angioedema and chronic urticaria into 2 separate groups.

Last, we must mention that there is a contradiction between our data regarding the prevalence of atopic diseases in the general population and previous studies that were conducted in Israel [24-26]. A possible explanation can be that our data were based only on the population eligible for military service. There are no data on patients who emigrated from Israel at an early age, died of the illness or were conscientious objectors.

While more facts are being elucidated, the exact pathophysiology of KD remains an enigma. The unidentified infectious agents, the strong genetic predilection even though low familial incidence rates are reported as well as the clear relationship with atopic diseases in KD cases and in their family members, all point towards a multifactorial mechanism. Further research is required for better understanding of this disease. The caring physician should be alert to the link between $\mathrm{KD}$ and allergic diseases, and conduct proper follow-up and tests as needed.

\section{References}

1 Kawasaki T, Kosaki F, Okawa S, Shigematsu I, Yanagawa $\mathrm{H}$ : A new infantile acute febrile mucocutaneous lymph node syndrome (MLNS) prevailing in Japan. Pediatrics 1974; 54:271-276.

2 Kato H, Sugimura T, Akagi T, Sato N, Hashino K, Maeno Y, et al: Long-term consequences of Kawasaki disease. A 10- to 21-year follow-up study of 594 patients. Circulation 1996;94:1379-1385.

3 Dajani AS, Taubert KA, Gerber MA, Shulman ST, Ferrieri P, Freed M, et al: Diagnosis and therapy of Kawasaki disease in children. Circulation 1993;87:1776-1780.

4 Taubert KA, Rowley AH, Shulman ST: Nationwide survey of Kawasaki disease and acute rheumatic fever. J Pediatr 1991;119: 279-282.

5 Holve TJ, Patel A, Chau Q, Marks AR, Meadows A, et al: Long-term cardiovascular outcomes in survivors of Kawasaki disease. Pediatrics 2014;133:305-311.

6 Nakamura Y, Yashiro M, Uehara R, Sadakane A, Tsuboi S, Aoyama Y, et al: Epidemiologic features of Kawasaki disease in Japan: results of the 2009-2010 nationwide survey. J Epidemiol 2012;22:216-221.

7 Kuo HC, Yang KD, Juo SH, Liang CD, Chen WC, Wang YS, et al: ITPKC single nucleotide polymorphism associated with Kawasaki disease in a Taiwanese population. PLoS One 2011;6:e17370.

8 Du ZD, Zhao D, Du J, Zhang YL, Lin Y, Liu C, et al: Epidemiologic study on Kawasaki disease in Beijing from 2000 through 2004. Pediatr Infect Dis J 2007;26:449-451.

9 Grunebaum E, Blank M, Cohen S, Afek A, Kopolovic J, Meroni PL, et al: The role of antiendothelial cell antibodies in Kawasaki disease - in vitro and in vivo studies. Clin Exp Immunol 2002;130:233-240.
10 Bar-Meir M, Haklai Z, Dor M: Kawasaki disease in Israel. Pediatr Infect Dis J 2011;30: 589-592.

11 Tacke CE, Breunis WB, Pereira RR, Breur JM, Kuipers IM, Kuijpers TW: Five years of Kawasaki disease in the Netherlands: a national surveillance study. Pediatr Infect Dis J 2014; 33:793-797.

12 Tsai YJ, Lin CH, Fu LS, Fu YC, Lin MC, Jan SL: The association between Kawasaki disease and allergic diseases, from infancy to school age. Allergy Asthma Proc 2013;34:467-472.

13 Kuo HC, Chang WC, Yang KD, Yu HR, Wang CL, Ho SC, et al: Kawasaki disease and subsequent risk of allergic diseases: a populationbased matched cohort study. BMC Pediatr 2013;13:38.

14 Liew WK, Lim CW, Tan TH, Wong KY, Tai BC, Quek SC, et al: The effect of Kawasaki disease on childhood allergies - a sibling control study. Pediatr Allergy Immunol 2011;22:488493.

15 Webster RJ, Carter KW, Warrington NM, Loh AM, Zaloumis S, Kuijpers TW, et al: Hospitalization with infection, asthma and allergy in Kawasaki disease patients and their familes: genealogical analysis using linked population data. PLoS One 2001;6:e28004.

16 Wei CC, Lin CL, Kao CH, Liao YH, Shen TC, Tsai JD, et al: Increased risk of Kawasaki disease in children with common allergic diseases. Ann Epidemiol 2014;24:340-343.

17 Lee SB, Kim YH, Hyun MC, et al: T-helper cytokine profiles in patients with Kawasaki disease. Korean Circ J 2015;45:516-521.

18 Matsuoka S, Tatara K, Nakagawa R, Mori K, Kuroda Y: Tendency toward atopy in Kawasaki disease. Eur J Pediatr 1997;156:30-32.
19 Sato S, Kawashima H, Kashiwagi Y, Fujioka T, Takekuma K, Hoshika A: Association of mannose-binding lectin gene polymorphisms with Kawasaki disease in the Japanese. Int J Rheum Dis 2009;12:307-310.

20 Worthley DL, Bardy PG, Mullighan CG: Mannose-binding lectin: biology and clinical implications. Intern Med J 2005;35:548-555.

21 Kuo HC, Yang KD, Liang CD, Bong CN, Yu HR, Wang L, et al: The relationship of eosinophilia to intravenous immunoglobulin treatment failure in Kawasaki disease. Pediatr Allergy Immunol 2007;18:354-359.

22 Biezeveld MH, van Mierlo G, Lutter R, Kuipers IM, Dekker T, Hack CE, et al: Sustained activation of neutrophils in the course of $\mathrm{Ka}$ wasaki disease: an association with matrix metalloproteinases. Clin Exp Immunol 2005; 141:183-188.

23 Gualano RC, Vlahos R, Anderson GP: What is the contribution of respiratory viruses and lung proteases to airway remodeling in asthma and chronic obstructive pulmonary disease? Pulm Pharmacol Ther 2006;19:18-23.

24 Shohat T, Golan G, Tamir R, Green MS, Livne I, Davidson Y, Harari G, Garty BZ: Prevalence of asthma in 13-14 year-old schoolchildren across Israel. Eur Respir J 2000;15:725-729.

25 Cohen S, Berkman N, Avital A, Springer C, Kordoba L, Haklai Z, Eshel A, Goldberg S, Picard E: Decline in asthma prevalence and severity in Israel over a 10-year period. Respiration 2015;89:27-32.

26 Graif Y, Garty BZ, Livne I, Green MS, Shohat $\mathrm{T}$ : Prevalence and risk factors for allergic rhinitis and atopic eczema among schoolchildren in Israel: results from a national study. Ann Allergy Asthma Immunol 2004;92:245249 